\title{
Manejo interdisciplinario de la visión baja por microftalmos
} Reporte de un caso

\author{
Salas-Hernández Luis Héctor*
}

\section{Resumen}

- La visión baja es un problema visual donde el paciente ex-

- perimenta disminución en la agudeza visual que no puede

- mejorarse con un anteojo convencional ó pérdida en el campo

- visual a causa de una enfermedad ocular de origen degenera-

- tivo e irreversible en la mayoría de los casos. El microftalmos es

- una anomalía ocular en la que uno, o ambos globos oculares,

- tienen una notoria reducción del diámetro del globo ocular

- y puede estar asociado a coloboma, aniridia, hipermetropía

- elevada, glaucoma y presentar otras anomalías, como: micro-

- cornea y hendidura palpebral estrecha, leucoma o catarata.

- Caso clínico: paciente femenino de 40 años de edad, ama de

- casa, en aparente endotropia de ojo derecho con componen-

- te vertical. Al nacer le diagnosticaron microftalmos, catarata

- congénita en ambos ojos, a los 30 años le diagnostican glau-

- coma primario de ángulo cerrado asociado al microftalmos. La

- estrategia de manejo propuesta fue el uso de ayuda óptica

- convencional y no convencional, citas subsecuentes para reha-

- bilitación con ayuda óptica no convencional, se indica uso de

- bastón largo y técnicas de orientación y movilidad. Conclusión:

- el trabajo multidisciplinario entre el oftalmólogo y el optome-

- trista es fundamental, el mejor tratamiento siempre será la

- prevención ya que algunas enfermedades del globo ocular

- pueden llegar a ser irreversibles. La preparación del optome-

- trista en esta área es fundamental para ofrecer tratamientos

- a los pacientes con visión baja, ya que se requiere del manejo

- de cartillas especiales para agudeza visual y cálculos específ-

- cos para la adaptación de las ayudas ópticas no convenciona-

- les. LUX MÉDICA, AÑ0 9, NÚMERO 26, ENERO-ABRIL 2014, PP 43-49.

\section{Abstract}

Low vision is a visual problem where the patient presents a decrease in visual acuity that cannot improve it with a conventional eyeglasses. The loss of the visual field is caused by in most cases irreversible degenerative eye disease.The microphtalmos is an ocular anomaly in which one, or both eyes, have a marked reduction of the diameter of the eye and may be associated with coloboma, aniridia, retinal dysplasia, high hyeropia , glaucoma, and present other anomalies, such as: microcornea and narrow lid aperture, leucoma or cataract. Case report: female patient of 40 years old, housewife, in apparent esotropia of right eye with vertical component. At birth she was diagnosed with microphthalmos, congenital cataract in both eyes, at age 30 was diagnosed with primary closedangle glaucoma associated with microphthalmos. Proposed management plan was the use of conventional and non-conventional optical aids, subsequent appointments to rehabilitation with non-conventional optical assistance, use of the long cane and orientation and mobility techniques. Conclusion:The multidisciplinary work between ophthalmologist and optometrist is essential, the best treatment will always be prevention, because some ocular diseases can become irreversible, it is essential that the optometrist is trained in this area to offer treatments to patients with low vision, since it requires the use of special visual acuity charts and specific calculations for the prescription of non- conventional optical aids. LUXMÉDICA, AÑO 9, NÚMERO 26, ENERO-ABRIL 2014, PP 43-49.

Maestro en Ciencias Biomédicas. Profesor investigador del Departamento de Optometría del Centro de Ciencias de la Salud de la Universidad Autónoma de Aguascalientes.

Fecha de recibido: 3 marzo 2014

Fecha de aceptación: 10 de marzo 2014

Correspondencia: MCB. Luis Héctor Salas Hernández. UMD edificio 101 planta alta. Av universidad \#940. C.p. 20131 Aguascalientes, Ags., México. Tel 01(449) 910 8441. Correo electrónico Ihsalas@correo.uaa.mx 


\section{Introducción}

Se considera debilidad visual cuando una persona presenta un daño completo o una limitación significativa de la función visual resultante de una enfermedad, trauma o condición congénita y no se puede mejorar con corrección óptica convencional, medicación o cirugía1. La debilidad visual es un problema de salud por el cual en los últimos tiempos, los diferentes organismos de salud mundial trabajan para que disminuya el número de personas que la padecen. Los pacientes con debilidad visual se puede dividir en dos grandes grupos: pacientes que cursan con ceguera y pacientes con visión baja. La ceguera es la ausencia por completo de la función visual y la visión baja, antes llamada visión subnormal, es un problema visual donde el paciente experimenta disminución en la agudeza visual y pérdida en el campo visual a causa de una enfermedad ocular de origen degenerativo e irreversible en la mayoría de los casos. También se asocia a enfermedades sistémicas como la hipertensión la diabetes. Si bien la visión baja no es considerada una enfermedad sí es el resultado de una de ellas².

Esta disminución de agudeza y la pérdida en el campo visual no puede ser corregida con el uso de ayudas ópticas convencionales, como lentes de armazón o lentes de contacto. El tratamiento de la visión baja consiste en ampliar el tamaño de la imagen que se produce en la retina, mediante sistemas de ampliación. De esta manera se estimulan más células retinianas y se envía mayor información al cerebro para interpretar la imagen. La ampliación se determina después de haber compensado el estado refractivo de lejos del paciente. Los cinco métodos de la amplificación son: ampliación relativa al tamaño, relativa a la distancia, angular, ampliación por proyección, ampliación total. También se basa en rehabilitar el resto visual del paciente con microscopios, lupas, telemicroscopios, telescopios ó circuitos cerrados de televisión, que son considerados como ayudas ópticas no convencionales².

La mayoría de los pacientes con debilidad visual cursan también con problemas de tipo psicosocial y el rechazo de la sociedad. La falta de una legislatura para discapacitados y la escasa educación que se tiene para interactuar con una persona no solo débil visual, si no con algún otro tipo de discapacidad, hace que las personas discapacitadas se alejen y se marginen de la vida cotidiana. ${ }^{3-4}$

A un paciente que cursa con ceguera se le puede rehabilitar con técnicas de ambulación o su modalidad con bastón, practicando la escritura y la lectura mediante el sistema Braille, así como las técnicas de seguimiento y guía. A un paciente con visión baja se le rehabilita mediante el uso de ayudas ópticas no convencionales partiendo de la emetropización. Con ayudas no ópticas como el 
uso del bastón largo, tiposcopios y macrotipos, y las técnicas de orientación y movilidad ( $\mathrm{O}$ y $\mathrm{M}$ ). En ambos casos, la meta de la rehabilitación es reincorporar al paciente a la sociedad y lograr la mayor independencia dentro de lo que sea posible $e^{2-5}$.

La Organización Mundial de la Salud (OMS) considera que existe ceguera legal cuando la visión es menor de 20/200 en el mejor ojo y con la mejor corrección teniendo un campo visual inferior a $20^{\circ}$.

Clínicamente se identifica un paciente con visión baja cuando su mejor agudeza visual alcanzada es de 20/80 con su mejor corrección y un campo visual igual o menor a $20^{\circ}$ y esto le impida al paciente realizar actividades de la vida cotidiana.

La visión baja se clasifica de acuerdo a su agudeza visual en moderada, severa y profunda según August Colenbrander ${ }^{2-6}$. (Tabla 1)

\section{Tabla I}

\section{Clasificación de la baja visión por agudeza visual}

\begin{tabular}{|ccc|}
\hline MODERADA & SEVERA & PROFUNDA \\
\hline $20 / 80$ & $20 / 100$ & $20 / 125$ \\
\hline $20 / 160$ & $20 / 200$ & $20 / 250$ \\
\hline $20 / 320$ & $20 / 400$ & $20 / 500$ \\
\hline $20 / 630$ & $20 / 800$ & $20 / 1000$ \\
\hline & & Hasta proyección de luz \\
\hline
\end{tabular}

El microftalmos es una anomalía ocular en la que uno o ambos globos oculares, tienen una notoria reducción del diámetro del globo ocular. Las personas con microftalmos pueden cursar con un coloboma, aniridia, displasia retiniana, hipermetropía elevada, glaucoma y tener otras anomalías, como: microcórnea y hendidura palpebral estrecha, opacificación de córnea o del cristalino (catarata) ${ }^{7-8}$. El principal síntoma que origina la catarata es la pérdida progresiva de la agudeza visual. Además, se produce una disminución de la sensibilidad a los contrastes y una alteración en la apreciación de los colores ${ }^{9}$. Se puede cursar con microftalmos por trastornos alimenticios acompañados por agentes teratógenos carencia o exceso de vitamina $A$, rubéola y anomalías sistémicas. El globo ocular y la córnea son congénitamente pequeños. Existe generalmente un desarrollo anormal del cristalino el cual puede ser esférico, cataratoso o estar ausente ${ }^{10-11 .}$

El glaucoma de ángulo cerrado se presenta cuando el ángulo irido-corneal tiene una forma estrecha y esta forma impide la circulación del humor acuoso. El iris queda comprimido contra el cristalino; a medida que la presión en la cámara posterior aumenta, el iris se abomba hacia adelante, obstruyendo el ángulo de filtración y contribuyendo aun más al incremento de la presión intraocular. El glaucoma de ángulo cerrado generalmente se acompaña por: dolor ocular, hiperemia, lagrimeo, fotofobia y percepción de halos de colores por el edema corneal ${ }^{11-12}$. 


\section{Presentación del caso clínico}

Se trata de paciente femenino de 40 años de edad, ama de casa, en aparente endotropia de ojo derecho con componente vertical. Al nacer le diagnosticaron microftalmos, catarata congénita en ambos ojos, a los 30 años fue diagnosticada con glaucoma primario de ángulo cerrado asociado al microftalmos, tratado con latanaprost y timolol. En la última revisión con su médico oftalmólogo se pudo observar que las cataratas habían sido absorbidas. Con su última corrección óptica prescrita hace un año, refiere que no hay ninguna mejoría. Acude a la clínica de baja visión, donde fue evaluada y se encontró lo siguiente: diagnóstico refractivo, hipermetropía y presbicie. La corrección óptica en uso es ojo derecho: +6.25 dioptrías, en ojo izquierdo + 10.00 dioptrías (Imagen 1)

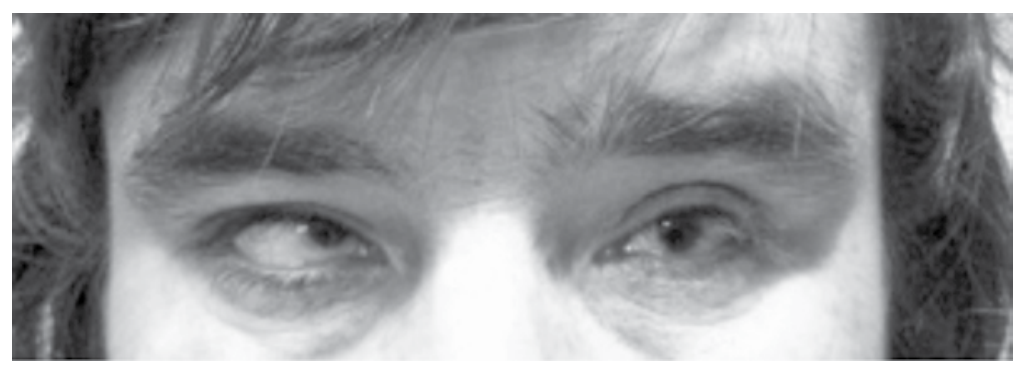

Imagen 1. Paciente con microftalmos, endotropia con componente vertical en posición primaria de mirada.

La paciente expresa que requiere mejorar la visión lejana, poder leer en visión próxima, lograr un mejor desplazamiento tanto en interiores como en exteriores y de ser posible volver a realizar algunas actividades de la vida cotidiana como cocinar, ver la televisión, caminar y bordar. La paciente se desplaza por tacto y es acompañada de algún familiar, utiliza filtro solar.

Los resultados de la perimetría revelan la presencia de escotomas relativos centrales y periféricos en ojo derecho. Escotomas relativos periféricos en ojo izquierdo. La presión intraocular registrada en ambos ojos fue de $20 \mathrm{~mm} \mathrm{Hg}$.

La agudeza visual en visión lejana sin corrección fue de 20/360 visión excéntrica movimiento nasal, visión temporal (M:N$\mathrm{V}: \mathrm{T}$ ) y en ojo izquierdo 20/200 con la cartilla Feinbloom a 3mts. La agudeza visual cercana sin corrección para ojo derecho fue de 20/200 visión excéntrica M:N- V:T y para ojo izquierdo 20/200 con la cartilla New ETDRS a $25 \mathrm{~cm}$.

En cuanto a la agudeza visual con corrección, para ojo derecho fue de 20/400 visión excéntrica $M: N-V: T$. y para ojo izquierdo 20/100 -1 con la cartilla Feinbloom a 3mts. En visión cercana la agudeza obtenida con corrección fue de 20/200 visión excéntrica $M: N-V: T$. y en ojo izquierdo 20/100 New ETDRS a $25 \mathrm{~cm}$.

El primer objetivo para el tratamiento fue prescribir la mejor corrección ópti- 
ca convencional. Ojo derecho: $+10.75=$ $-0.75 \times 0^{\circ}$ dioptrías ojo Izquierdo $+14: 50$ $=-1.00 \times 115^{\circ}$ dioptrías con la cual se obtuvo una agudeza visual de lejos de 20/80 para cada ojo con la cartilla Feinbloom a 3 mts. Adición de +1.50 para visión cercana, logrando una agudeza visual para ojo derecho de 20/80 y de 20/70 para el ojo izquierdo con la cartilla New ETDRS a $25 \mathrm{~cm}$.

El segundo objetivo del tratamiento fue proporcionar una ayuda óptica no convencional para lo cual se calculó para visión lejana un telescopio binocular de $3 \mathrm{X}$ aumentos mejorando la agudeza visual para ojo derecho de 20/30 visión excéntrica M:N- V:T. Para ojo izquierdo de Ol: 20/20 New ETDRS a 6mts. En cuanto a la visión cercana: Ms 1.5X aumentos OD: 20/30 visión excéntrica M:N- V:T. OI: 20/20 New ETDRS a $16.66 \mathrm{~cm}$ DT. Con la finalidad de proporcionar una mejor protección en exteriores se prescribió un filtro añadido sobre la corrección óptica de Glarecutter 511. Finalmente se propuso acudir a sesiones de rehabilitación y asesoría para el uso de la ayuda óptica no convencional, uso de bastón largo y técnicas de orientación y movilidad.

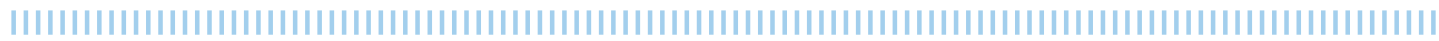

\section{Discusión}

En el mundo hay aproximadamente 285 millones de personas con discapacidad visual, de las cuales 39 millones son ciegas y 246 millones presentan visión baja.

Aproximadamente un $90 \%$ de la carga mundial de discapacidad visual se concentra en los países en desarrollo. El $82 \%$ de las personas que padecen ceguera tienen 50 años o más. En términos mundiales, los errores de refracción no compensados constituyen la causa más importante de discapacidad visual, pero en los países de ingresos medios y bajos las cataratas siguen siendo la principal causa de ceguera reversible. El número de personas con discapacidades visuales atribuibles a enfermedades infecciosas ha disminuido considerablemente en los últimos 20 años. El $80 \%$ del total mundial de casos de discapacidad visual se pueden evitar o curar ${ }^{13}$.

Para dar inicio al tratamiento, primeramente se requiere prescribir la corrección óptica convencional, ya que es indispensable partir de la emetropización para poder utilizar ayudas ópticas no convencionales ${ }^{14}$.

El microscopio y el telescopio, los cuales se adaptan en el ojo que obtiene una mejor agudeza visual y en posición primaria de mirada, ya que es difícil lograr la fusión de imágenes debido a la aparente endotropia derecha con componente vertical y a la visión excéntrica que presenta movimiento nasal, visión temporal.

Se realizaron pruebas con el microscopio (Ms) y telescopio (Ts) de manera binocular para alternar la visión, pero la paciente refirió mayor cansancio y prefirió utilizarlo de manera monocular. El filtro se añadió a su corrección óptica convencional. Se prescribieron dos pares de anteojos para visión lejana, uno sin filtro para interior y otro con filtro para utilizarse en exteriores.

La rehabilitación se llevó a cabo en visión lejana con el telescopio Ts monocular en ojo izquierdo, con ejercicios de seguimiento y ubicación de objetos. En visión cercana con el microscopio Ms logrando una excelente velocidad de lectura leyendo de 40 a 50 palabras por minuto de manera monocular. Utilizando el ojo izquierdo, con la cartilla de lectura Colenbrander. También se rehabilitó al paciente con técnicas de orientación y movilidad (O y M). El bastón largo se recomendó con el uso de la ayuda óptica convencional y no convencional enseñando al paciente técnicas de desplazamiento con bastón. Después de cuatro sesiones se dio de alta al paciente pues 
se cumplieron las metas de lectura que es un mínimo de 30 palabras por minuto. La práctica con el bastón largo se enfatizó en la ambulatoria comenzando con recorridos cortos con ayuda óptica hasta llegar a recorridos largos y con el telescopio.

\section{Conclusiones}

La visión baja es una discapacidad visual que no respeta ni edad, ni género y son pocos los profesionales de la salud visual que se dedican a su tratamiento. La preparación del optometrista es fundamental para ofrecer tratamientos a los pacientes con visión baja, pues se requiere del manejo de cartillas especiales y cálculos específicos para la adaptación de las ayudas ópticas no convencionales. El trabajo entre el oftalmólogo y el optometrista es clave para la ayuda de estos pacientes. El oftalmólogo es el encargado de diagnosticar y emitir un tratamiento, dar seguimiento a la enfermedad ocular. Una vez tratada la enfermedad ocular, el oftalmólogo debe canalizar al paciente con el optometrista para que sea evaluada la función visual y el estado refractivo. Si el paciente no cursa con visión baja, su tratamiento se basará en la adaptación de ayudas ópticas convencionales. Si el optometrista identifica que el paciente cursa con visión baja, el tratamiento a ofrecer será por medio de ayudas ópticas no convencionales y ayudas no ópticas para reforzar la movilidad y orientación del paciente. Por esa razón los profesionales de la salud visual, optometrista y oftalmólogo, deberán trabajar de manera conjunta para ofrecer a la población un tratamiento de primer nivel. El mejor tratamiento siempre será la prevención a través de visitas periódicas al profesional de la salud visual, y revisiones médicas por lo menos una vez al año. Lo anterior ayudará a tener una mejor cultura en salud ya que algunas enfermedades del globo ocular pueden llegar a ser irreversibles como el caso de la microftalmos. La ayuda que brinda el profesional de la salud visual no sólo debe ser basada en tratamientos médicos u ópticos, sino también, rehabilitar al paciente dentro de lo que sea posible para que pueda llevar a cabo actividades de la vida cotidiana y reinsertarlo a la sociedad.

\section{Bibliografía}

1. Eleanor E. Faye y otros. Manual de entrenamiento en la atención de la baja visión. EU. Lighthouse. 2003; 1(2): $4-6$.

2. Barañano Ángel, Formación en la Baja Visión. Madrid España. Edit AVS 2011; 1(10): 7 - 21.

3. Tay KC, Drury VB, Mackey S. The role of intrinsic motivation in a group of low vision patients participating in a self-management programme to enhance self-efficacy and quality of life. Int J Nurs Pract. 2014; 20(1):17-24.

4. Perla Catherine Mayo. Inducción a la Baja visión. Argentina Edit Bastón Verde. 2011;1 (1) 34 - 36.

5. Nau AC, Pintar C, Fisher C, Jeong JH, Jeong K. A standardized obstacle course for assessment of visual function in ultra low vision and artificial vision.JVisExp.2014;10(84):5

6. Splendiani B, Ribera M, Garcia R, Termens M..Do Physicians Make Their Articles Readable for Their Blind or Low-Vision Patients? An Analysis of Current Image Processing Practices in Biomedical Journals from the Point of View of Accessibility. J Digit Imaging. 2014; 3(7) 31-28.

7. Toto L, Di Antonio L, Mastropasqua A, De Nicola C, Mastropasqua L.Rehabilitation with MP1 biofeedback training of a posterior microphthalmos case. Can J Ophthalmol. 2013 Oct;48(5):e107-11.

8. Huo LA, Yang J, Zhang C. Regional difference of genetic factors for congenitals cataract. The results of congenital cataract screening under normal pupil 
conditions for infants in Tianjin city. Eur Rev Med Pharmacol Sci. 2014;18(3):426-30

9. Li DM. Give attention to standardized management of orbital development in Chinese with microphthalmos or anophthalmos. Zhonghua Yan Ke Za Zhi. 2013; 49(8):676-8.

10. Sidoff $A E$, Garza L, Batlle J, Bordlee B, Palacios E, Neitzschman HR. Radiology case of the month. An infant with leukocoria: persistent fetal vasculature syndrome. Persistent fetal vasculature syndrome (PFVS) of the right eye associated with microphthalmia, bilateral optic nerve and optic chiasm hypoplasia, and absence of the neurohypophysis. J La State Med Soc. 2013 May-Jun;165(3):168-70

11. Skalicky SE, White AJ, Grigg JR, Martin F, Smith J, Jones $M$, Donaldson $C$, Smith JE, Flaherty $M$, Jamieson RV. Microphthalmia, anophthalmia, and coloboma and associated ocular and systemic features: un- derstanding the spectrum. JAMA Ophthalmol. 2013 Dec; 131(12):1517-24.

12. Kothari R, Tathe S, Gogri P, Bhandari A. Lens-Induced Glaucoma: The Need to Spread Awareness about Early Management of Cataract among Rural Population. ISRN Ophthalmol. 2013 Jun; 25(13):5157

13. Organización Panamericana de Salud, Uruguay $2013 \quad \mathrm{http}: / / w w w . p a h o . o r g / u r u /$ index.php?option $=$ com_content \&vie $w=$ article \&id $=770:$ ceguera-y-discapacidad-visual \&catid $=704:$ discapacidad accesibilidad\&itemid $=247$

14. Quang Do V, Li R, Ma M, Pooley C, Trinh C, Peattie L, Palagyi A, McCluskey P, Keay L. Investigating cataract referral practices used by Australian optometrists. Clin Exp Optom. 2014; 4(3):10 - 11 